\title{
The effect of marker size on the perception of an empty interval
}

\author{
FUMINORI ONO \\ Juntendo University School of Medicine, Tokyo, Japan \\ AND \\ Shigeru KitaZaWa \\ Juntendo University School of Medicine, Tokyo, Japan \\ and CREST, Japan Science and Technology Agency, Saitama, Japan
}

\begin{abstract}
Research shows that the time that is spent perceiving a brief visual stimulus is experienced as increasing as the size of the stimulus increases. We examined whether the experienced duration of time that is spent attending the perception of an empty interval — demarcated by the offset of one marker and the onset of a second markerdepends on the size of the markers themselves. Previous theories predict that the perceived time that is spent viewing offset-to-onset intervals decreases as the size of the markers increases, and that the perceived time that is spent viewing the markers increases. We demonstrated that empty intervals between the presentations of large markers were perceived to be longer in duration than those occurring between the presentations of small markers, and that the second marker was critical to this effect of physical size on apparent duration. We report that the size effect disappeared when the interval was filled with the presentation of a circle, and we conclude that the intensity of the second marker altered perceptions in an empty-interval-specific manner.
\end{abstract}

Substantial evidence indicates that subjective perceptions of time do not exactly match the actual duration of an event; rather, perceived durations stretch or contract depending on properties that are ostensibly independent of elapsed time. For example, the perceived durations of briefly presented stimuli increase as the area size (Long \& Beaton, 1980; Thomas \& Cantor, 1975), number (Mo, 1975), or complexity (Schiffman \& Bobko, 1974) of such stimuli increases. Researchers have explained the changes in time perception in terms of some kind of internal clock (Treisman, 1963) that regulates judgments of time spans. This internal clock, it has been suggested, becomes faster in response to the attention or arousal that is evoked by perceptions of more numerous, larger, and/or more complex stimuli. This increased clock speed leads to an increased number of counts in a given period, which eventually leads to elongated perception of time.

Thus far, studies have focused primarily on the perception of elapsed time that is filled with stimuli; perceptions of time that is not filled with stimuli should prove equally important to our understanding of many kinds of behaviors that require exact timing (e.g., cooking, sports, or playing music). The present study investigates how humans perceive the duration of time during an empty interval (i.e., one that is not filled with stimuli) that is separated by two markers indicating the beginning (the offset of the first marker) and the end (the onset of the second marker) of the interval. According to the internal-marker hypothesis (Grondin, 1993) - one of the most dominant in the field - internal markers are determined by the perceptions of the offset of the first stimulus and the onset of the second stimulus (Grondin, Ivry, Franz, Perreault, \& Metthé, 1996; Tse \& Penney, 2006). According to the internal-marker hypothesis, experimental manipulation can change the perceived duration of time that is spent viewing an empty interval, so that the perceived duration becomes shorter when the perception of the offset of the first stimulus is delayed or when the perception of the onset of the second stimulus is accelerated.

We examined the effect of the size of markers on the perceived duration of the time that is used to view an empty area between the markers; we expected the sizes of the markers to affect perceptions about offset and onset. On the basis of findings that the perceived duration of time that is required to view a visual stimulus increases as the size of that stimulus increases (Long \& Beaton, 1980; Thomas \& Cantor, 1975), we hypothesized that the perceived offset of a large first marker would be delayed in comparison with that of a small marker, even when the actual time of marker exposure remained constant. In addition, perception of stimulus onset has been reported to occur sooner when the intensity of a given stimulus is increased (Piéron, 1952; Pins \& Bonnet, 1996; Stafford $\&$ Gurney, 2004). The perceived onset of a large second

F. Ono, fuminori@juntendo.ac.jp 
marker, therefore, should occur sooner than that of a small second marker. The internal-marker hypothesis predicts that an empty interval that is sandwiched between large markers will be perceived as shorter than one that is sandwiched between small markers.

To test whether the time perception of an empty interval depends on the size of markers, we carried out seven experiments. In the first experiment, the two markers were either both large or both small (Figure 1A). In the second experiment, we tested whether the size of the first marker was responsible for the size effect by replacing the second marker with a cross (Figure 1C). In the third experiment, we examined the size effect of the second marker by replacing the first marker with a cross (Figure 1E). The fourth experiment tested the possibility that the participants had included the duration of the second marker in their estimation of the interval length. For this purpose, the second marker was presented until participants pressed the key for rating (Figure 1G). The fifth experiment tested whether the marker-size effect was obtained in the perception of an empty interval only. To examine this, the empty interval between the first and second markers was filled with a red circle (Figure 1I). The sixth experiment reexamined the marker-size effect by using a temporaldiscrimination task (Figure 2A). The seventh experiment examined whether the marker-size effect was caused by a mislocation of the temporal endpoint, which was signaled by the onset of the second marker. To test this possibility, we conducted tests of temporal-order judgments involving the onset of either large or small stimuli (Figure 3A).

\section{EXPERIMENT 1}

\section{Method}

Participants. Ten paid volunteers participated. All participants had normal or corrected-to-normal visual acuity, and all were unaware of the purpose of the experiment. In accord with institutional guidelines, each participant gave informed consent prior to participation.

Apparatus, Stimuli, and Procedure. In a quiet, dark room, seated participants viewed a monitor (Iiyama HM204D, $100 \mathrm{~Hz}$ ) that was located approximately $60 \mathrm{~cm}$ in front of them. They were instructed to estimate the length of time that they spent viewing a short empty interval that was demarcated on each side by a visual marker (i.e., empty-interval estimation). No feedback was given in any part of these experiments. Experiments were run on a PC/AT-compatible computer, using MATLAB (MathWorks) with Psychophysics Toolbox extensions (Brainard, 1997).

In an empty interval estimation task, the participants estimated the length of time that they spent viewing a short, empty interval that was demarcated by two visual markers. The visual markers were black, filled circles on a gray background that were presented in the center of the monitor. The empty interval that was to be estimated started at the offset of the first marker and ended at the onset of the second marker.

Before each experiment, the participants completed 24 training trials in which they were exposed to four different empty intervals (lasting $100,200,300$, and $400 \mathrm{msec}$ ) in a pseudorandom order, and were trained to rate each interval on a scale from 1 (shortest) to 4 (longest). Participants initiated each training trial by pressing a space bar. Following a blank screen that lasted $500 \mathrm{msec}$, the first marker (a circle $2^{\circ}$ in diameter) was presented for $1,500 \mathrm{msec}$, followed by a blank screen (lasting 100, 200, 300, or $400 \mathrm{msec}$ ) and then the second marker (circle of $2^{\circ}, 1,500 \mathrm{msec}$ ). Participants rated the time that they had spent with the empty interval by pressing a number key from 1 (shortest) to 4 (longest). In test trials $(n=120)$, participants rated an empty interval of either 150 or $350 \mathrm{msec}$ according to this four-point scale.

In Experiment 1 (Figure 1A), both markers were small $\left(1^{\circ}\right)$ under one condition (the small-small condition), and large $\left(3^{\circ}\right)$ under another (the large-large condition).

\section{Results}

Experiment 1 focused on the effects of the size of the visual markers (Figure 1A). Both the first and second markers were small $\left(1^{\circ}\right)$ under one condition (smallsmall), and large $\left(3^{\circ}\right)$ under another (large-large). Contrary to our expectations, participants experienced the duration of time that they spent viewing the empty intervals as longer under the large-large condition (mean score $=$ 2.38; Figure 1B) than under the small-small condition (mean score $=2.21$ ). The mean scores for each duration under each condition of Experiments 1-5 are presented in Table 1. A two-way ANOVA (within-subjects design) showed that the main effect of the marker size [smallsmall or large-large; $F(1,9)=9.11, p<.05]$ and that of the duration of the empty interval [150 or $350 \mathrm{msec}$; $F(1,9)=250.02, p<.001]$ were both significant, but their interaction was not $[F(1,9)=2.46, p=.15]$. These results cannot be explained by the internal-marker hypothesis.

\section{EXPERIMENT 2}

\section{Method}

Participants. Ten paid volunteers participated. All participants had normal or corrected-to-normal visual acuity, and all were unaware of the purpose of the experiment. In accord with institutional guidelines, each participant gave informed consent prior to participation. None of the participants in Experiment 2 had participated in Experiment 1.

Apparatus, Stimuli, and Procedure. The apparatus and procedure were the same as those in Experiment 1. The stimuli were different, however. In Experiment 2 (Figure 1C), the second marker was replaced with a cross $\left(2^{\circ}\right)$ under both conditions.

\section{Results}

In Experiment 2, we tested whether the size of the first marker was responsible for the size effect (Figure 1C).

Table 1

Mean Scores of Interval Estimations

\begin{tabular}{|c|c|c|c|c|}
\hline & \multicolumn{4}{|c|}{ Duration } \\
\hline & \multicolumn{2}{|c|}{$150 \mathrm{msec}$} & \multicolumn{2}{|c|}{$350 \mathrm{msec}$} \\
\hline & $M$ & $S E$ & $M$ & $S E$ \\
\hline \multicolumn{5}{|l|}{ Experiment 1} \\
\hline Small-small & 1.39 & 0.06 & 3.03 & 0.09 \\
\hline Large-large & 1.52 & 0.07 & 3.24 & 0.10 \\
\hline \multicolumn{5}{|l|}{ Experiment 2} \\
\hline Small-control & 1.85 & 0.11 & 3.10 & 0.09 \\
\hline Large-control & 1.80 & 0.14 & 3.23 & 0.07 \\
\hline \multicolumn{5}{|l|}{ Experiment 3} \\
\hline Control-small & 2.00 & 0.18 & 2.82 & 0.09 \\
\hline Control-large & 2.15 & 0.17 & 3.06 & 0.08 \\
\hline \multicolumn{5}{|l|}{ Experiment 4} \\
\hline Small-small & 1.40 & 0.06 & 2.90 & 0.14 \\
\hline Large-large & 1.62 & 0.09 & 3.22 & 0.05 \\
\hline \multicolumn{5}{|l|}{ Experiment 5} \\
\hline Small-red-small & 1.44 & 0.09 & 3.17 & 0.14 \\
\hline Large-red-large & 1.35 & 0.09 & 3.03 & 0.12 \\
\hline
\end{tabular}


A

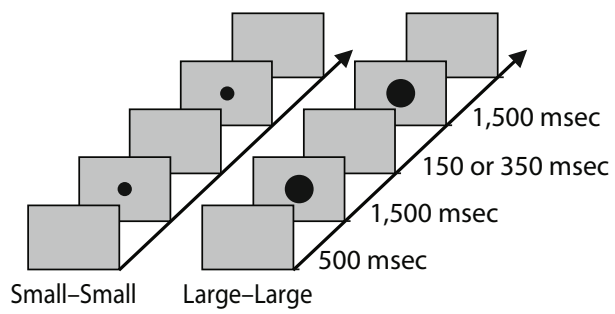

C

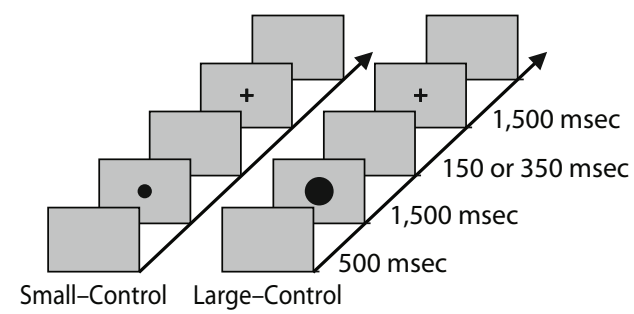

E

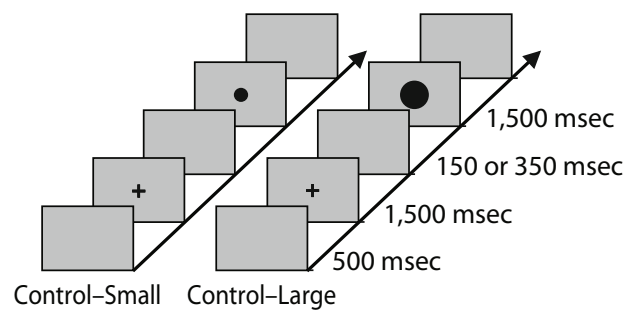

G

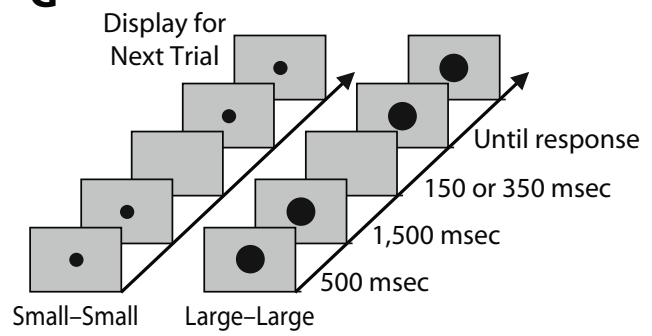

I

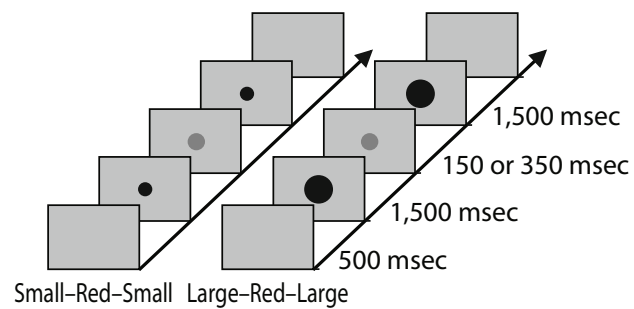

B

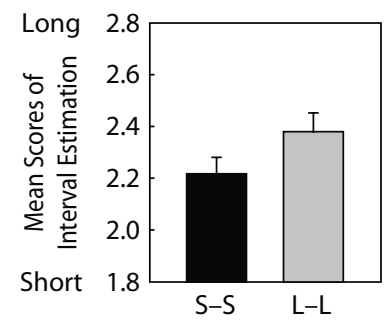

D

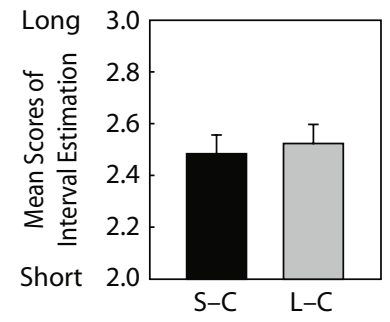

$\mathbf{F}$

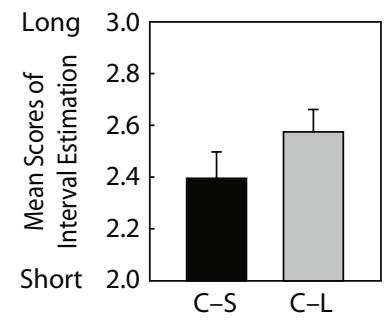

H

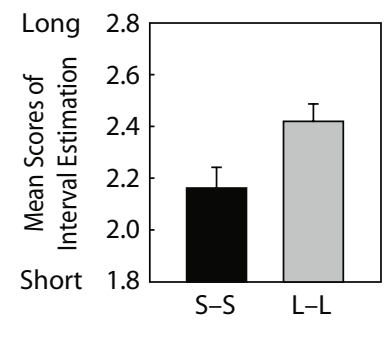

J

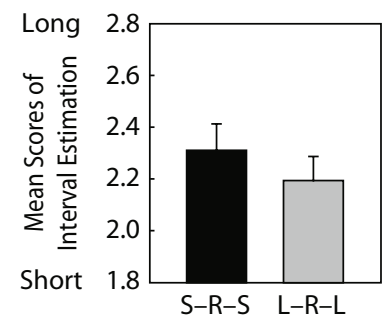

Figure 1. Effects of marker size on the estimation of an empty interval. Panels A, C, E, G, and $I$ show the size and shape of the first and second markers under two different conditions, and panels B, D, F, H, and J show the mean estimation score (on a scale of 1 to 4) under each condition. Error bars show the standard errors. In Experiment 1 (A, B), both markers were small under one condition (small-small) and large under another (large-large). In Experiment 2 (C, D), the first marker was small under one condition (small-control) and large under another (large-control). In Experiment 3 (E, F), the second marker was small in one condition (control-small) and large in another (large-control). In Experiment $4(G, H)$, the second marker was presented until the response. The first marker of the next trial was presented, without a gap, after the response. In Experiment 5 (I, J), the conditions were the same as those in Experiment 1, except that the empty interval was filled with a medium-sized red circle. 
Although the area size of the second marker was kept constant (intermediate cross: $2^{\circ}$ ), the first marker was small $\left(1^{\circ}\right)$ under one condition (small-control) and large $\left(3^{\circ}\right)$ under the other condition (large-control). The mean score of interval estimation was slightly longer under the largecontrol condition than under the small-control condition (by 0.04; Figure 1D); however, the two-way ANOVA failed to show significance in the main effect of the first marker size $[F(1,9)=0.71, p=.41]$. The main effect of the duration and the interaction between the size of the first marker and the duration were significant $[F(1,9)=$ $68.89, p<.001 ; F(1,9)=9.23, p<.05]$. Post hoc $t$ tests showed that the perceived time that was spent viewing the empty interval was significantly longer under the largecontrol condition than under the small-control condition when the empty interval was $350 \mathrm{msec}[t(9)=5.06$, $p<.05]$, but not when the empty interval was $150 \mathrm{msec}$ $[t(9)=0.62, p=.44]$. Although significant differences were observed in regard to the former condition, the mean difference in estimated interval durations between the large-control and the small-control conditions $(0.04)$ was not sufficient to explain the difference that was obtained between the large-large and the small-small conditions (0.17) in Experiment 1.

\section{EXPERIMENT 3}

\section{Method}

Participants. Ten paid volunteers participated. All participants had normal or corrected-to-normal visual acuity, and all were unaware of the purpose of the experiment. In accord with institutional guidelines, each participant gave informed consent prior to participation. None of the participants in Experiment 3 had participated in Experiments 1 and 2.

Apparatus, Stimuli, and Procedure. The apparatus and procedure were the same as those in Experiment 1. The stimuli were different, however. In Experiment 3 (Figure 1E), the first marker was replaced with a cross $\left(2^{\circ}\right)$ under both conditions.

\section{Results}

In Experiment 3, we tested whether the second marker was a dominant factor (Figure 1E). Although the first marker was kept constant (a cross of $2^{\circ}$ ), the size of the second marker was either small (control-small condition) or large (control-large condition). We found that the mean score was significantly longer under the control-large condition (2.57) than under the control-small condition (2.39). A two-way ANOVA showed that the main effect of the size of the second marker and that of the duration were significant [Figure 1F; $F(1,9)=7.77, p<.05 ; F(1,9)=$ $17.48, p<.01]$, but their interaction was not $[F(1,9)=$ $1.89, p=.20]$. The difference between the estimated scores that were obtained under the control-large condition and those that were obtained under the control-small condition (0.19) in Experiment 3 was as large as the difference between the estimated scores that were obtained under the large-large condition and those that were obtained under the small-small condition in Experiment 1 (0.17). The results of Experiments 1 and 2 clearly show that the size of the second marker was a dominant factor.

\section{EXPERIMENT 4}

\section{Method}

Participants. Eight paid volunteers participated. All participants had normal or corrected-to-normal visual acuity, and all were unaware of the purpose of the experiment. In accord with institutional guidelines, each participant gave informed consent prior to participation. None of the participants in Experiment 4 had participated in Experiments 1-3.

Apparatus, Stimuli, and Procedure. The apparatus and stimuli were the same as those in Experiment 1. The procedure was different, however. In Experiment 4 (Figure 1G), the second marker was presented until the participant pressed the rating key, and the first marker of the next trial was presented immediately after this keypress.

\section{Results}

In Experiment 4, we tested whether the effect of marker size was caused by the inadvertent addition of the duration that was spent viewing the second marker to the time that was spent viewing the empty interval. To prevent participants from adding the whole duration that was spent viewing the second marker, we presented the second marker until participants pressed the key for rating (Figure 1G). Under one condition, both markers were small (smallsmall condition), and under another, both were large (large-large condition). A two-way ANOVA showed that the main effect of the marker size and that of the duration were both significant [Figure 1H; $F(1,7)=10.80, p<$ $.05 ; F(1,7)=345.69, p<.001]$, but their interaction was not $[F(1,9)=0.47, p=.51]$. Accordingly, the perceived duration of the empty interval under the large-large condition (2.42) was longer than that under the small-small condition (2.15). The results of Experiment 4 replicated those of Experiment 1 and further eliminated the possibility that the marker-size effect was caused by an inadvertent inclusion of the entire duration of the second marker.

\section{EXPERIMENT 5}

\section{Method}

Participants. Nine paid volunteers participated. All participants had normal or corrected-to-normal visual acuity, and all were unaware of the purpose of the experiment. In accord with institutional guidelines, each participant gave informed consent prior to participation. None of the participants in Experiment 5 had participated in Experiments $1-4$.

Apparatus, Stimuli, and Procedure. The apparatus and procedure were the same as those in Experiment 1. The stimuli were different, however. In Experiment 5 (Figure 1I), the empty interval was filled with the presentation of a red circle $\left(2^{\circ}\right)$.

\section{Results}

In Experiment 5, we tested whether the effect of marker size was obtained only in relation to the perception of an empty interval. For this purpose, the empty interval between the first and second markers was filled with the presentation of a red circle (Figure 1I); this experiment was otherwise identical to the first experiment. A small black circle was presented before and after the red circle under one condition (small-red-small condition), and a large black circle was presented before and 
after the red circle (large-red-large condition) under another. In marked contrast to the results of previous experiments, the mean score under the small-red-small condition (2.31) was longer than that under the largered-large condition (2.19). A two-way ANOVA showed that the main effect of the marker size and that of the duration were significant [Figure 1J; $F(1,8)=6.84, p<$ $.05 ; F(1,8)=227.67, p<.001]$, but their interaction was not $[F(1,8)=0.44, p=.53]$. The results showed that the marker-size effect that is reported above is specific to the experience of the time that is spent in the perception of empty intervals versus time that is spent in the perception of filled intervals.

In Experiment 5, the apparent area size of the red circle might have been altered by the size-contrast illusion: A circle that is presented between large markers may appear to be smaller than an identically sized circle that is presented between two small markers (Cooper \& Weintraub, 1970). As a consequence, the perceived duration of the time that was devoted to viewing the red circle under the small-redsmall condition was longer than that under the large-redlarge condition (see also Ono \& Kawahara, 2007).

\section{EXPERIMENT 6}

\section{Method}

Participants. Eight paid volunteers participated. All participants had normal or corrected-to-normal visual acuity, and all were unaware of the purpose of the experiment. In accord with institutional guidelines, each participant gave informed consent prior to participation. None of the participants in Experiment 6 had participated in Experiments 1-5.

Apparatus, Stimuli, and Procedure. The apparatus was the same as that in Experiment 1. The stimuli and procedure were different, however.

In a temporal-discrimination task, participants specified the length of a short, empty interval by comparing it with a reference inter- val. One trial consisted of reference and test phases. In the reference phase, a cross $\left(2^{\circ}\right)$ was presented twice for $1,500 \mathrm{msec}$ per presentation, and the two presentations were separated by an empty interval of $250 \mathrm{msec}$ (Figure 2A). In the test phase, a circle ( $1^{\circ}$ under the small-small condition, and $3^{\circ}$ under the large-large condition) was presented twice, and the presentations were separated by an empty interval that was chosen pseudorandomly from eight intervals (150, $190,210,240,260,280,310$, or $350 \mathrm{msec}$ ). The participants were instructed to judge whether the empty interval in the test phase was longer than that in the preceding reference phase. Each participant completed 160 trials.

\section{Results}

In Experiment 6, we reexamined the effect of marker size on empty-interval perception by using a temporaldiscrimination task (Figure 2A). In this task, participants were asked to judge whether an empty interval that was sandwiched between two presentations of a small circle (small-small), or one that used large circles (large-large), was longer than a reference interval that was sandwiched between presentations of a cross. Note in Figure 2B that the proportion of "longer" judgments was generally larger under the large-large condition (filled circles) than under the small-small condition (open circles). The point of simultaneity, defined as the intersection of the sigmoid curve with the line that marks $P=0.5$, was smaller under the large-large condition ( $225 \mathrm{msec}$, solid curve) than under the small-small condition ( 249 msec, dotted curve). When the data were analyzed participant by participant, the mean of the point of simultaneity was significantly smaller under the large-large condition than under the small-small condition [paired $t$ test, $t(7)=3.21, p<$ $.05]$. A two-way ANOVA with two within-subjects variables revealed that the main effects of both the marker size (small-small or large-large) and the target interval (150, $190,210,240,260,280,310$, or $350 \mathrm{msec}$ ) were significant [Figure 2B; $F(1,7)=7.77, p<.05 ; F(7,49)=87.41$,
A

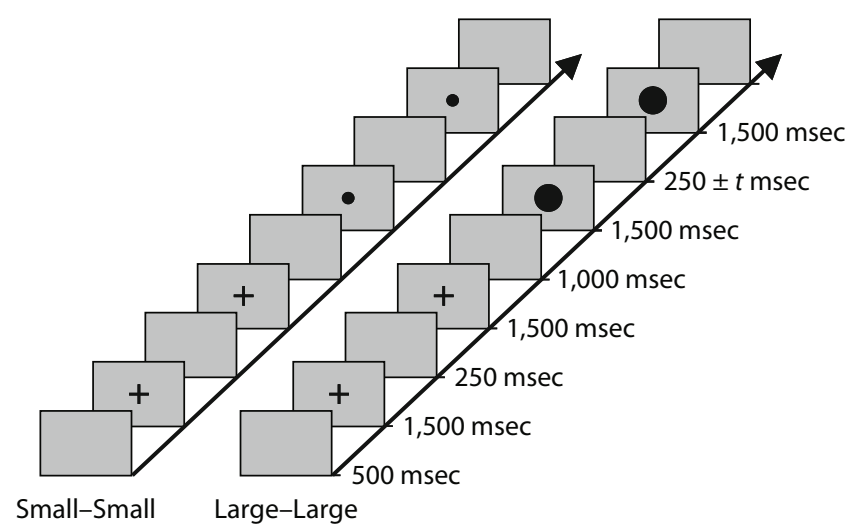

B

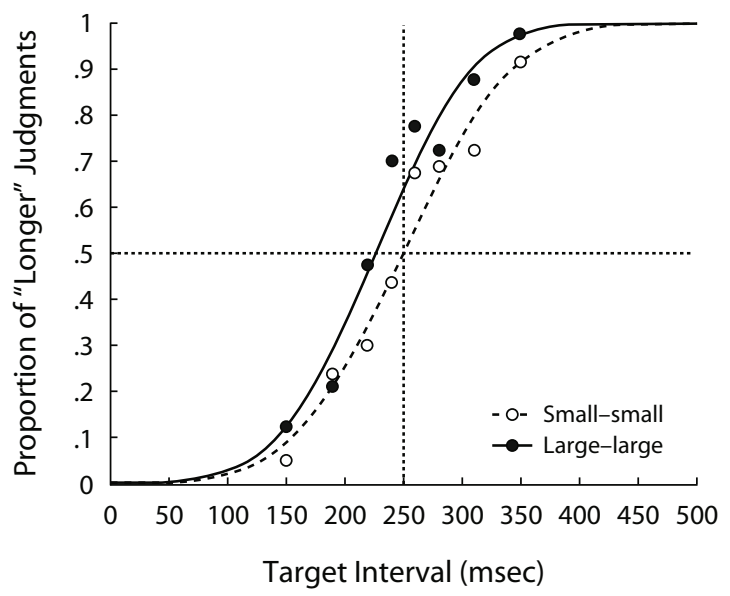

Figure 2. The marker-size effect was reexamined with a temporal-discrimination task (Experiment 6). (A) A sequence of events in one trial: Participants judged whether an empty interval that was demarcated by small circles (10 ${ }^{\circ}$, small-small) or by large circles (3', large-large) involved longer viewing durations than did a preceding reference empty interval that was demarcated by crosses (250 msec). (B) The probability that the participants judged that the empty interval was longer than that of the reference interval is plotted against the target interval. A vertical broken line at $250 \mathrm{msec}$ shows the length of the reference interval. Each symbol represents 80 judgments from 8 participants. 
$p<.001]$, but their interaction was not $[F(7,49)=0.92$, $p=.50]$. The results using temporal discrimination again showed that the empty interval was perceived as lasting longer when the marker size was larger.

\section{EXPERIMENT 7}

\section{Method}

Participants. Eight paid volunteers participated. All participants had normal or corrected-to-normal visual acuity, and all were unaware of the purpose of the experiment. In accord with institutional guidelines, each participant gave informed consent prior to participation. None of the participants in Experiment 7 had participated in Experiments 1-6.

Apparatus, Stimuli, and Procedure. The apparatus was the same as that in Experiment 1. The stimuli and procedure were different, however.

In a temporal-order judgment task, participants judged the order of two visual stimuli (a cross and a filled circle) that were presented in succession to the left and right of a fixed point at the center of the screen (Figure 3A). They responded by pressing the arrow key that corresponded to the side of the stimulus that the participant judged had been presented first. One of eight presentation intervals $(-100$, $-60,-30,-10,10,30,60$, or $100 \mathrm{msec}$ ) was assigned to each trial in a pseudorandom order. Positions (either left or right) of the stimuli were randomized across trials. A small circle $\left(1^{\circ}\right)$ and a cross $\left(2^{\circ}\right)$ were presented under one condition (small condition; Figure 3A, left) and a large circle $\left(3^{\circ}\right)$ and a cross $\left(2^{\circ}\right)$ were presented under another (large condition; Figure 3A, right). Each participant performed 80 trials under each condition.

\section{Results}

The results of Experiments 1-6 strongly suggest that the perception of an empty interval was affected by the size of the marker, especially that of the second marker. If the marker-size effect is caused by a mislocation of the temporal endpoint that is signaled by the onset of the second marker, the detection of spatially large stimuli will occur later than that of spatially smaller stimuli. To test this possibility, we conducted tests of temporal-order judgments concerning the onset of large and small stimuli (Figure 3A). A cross and a circle (large or small) were presented on the display, separated by a temporal lag, and the participants were asked to judge the order of stimuli. Figure 3B shows that marker size had little effect on the probability of "circle first" judgments. The points of simultaneity were almost identical under both conditions ( -5 and -3 msec under the large and small conditions, respectively). Their means were not significantly different $[t(7)=0.34, p=.74]$. A two-way ANOVA, with the area size (small or large) and interval $(-100,-60,-30,-10$, $10,30,60$, or $100 \mathrm{msec}$ ) as within-subjects variables, revealed that the main effect of the interval was significant [Figure 3B; $F(7,49)=212.83, p<.001]$, but the main effect of the area size was not $[F(1,7)=0.09, p=.77]$. Their interaction was not significant $[F(7,49)=0.49$, $p=.83]$. The results show that the detection of the small circle was as fast as that of the large circle. This finding clearly excludes the possibility that the marker-size effect resulted from either the delayed or the hurried detection of the second marker.

\section{DISCUSSION}

This study demonstrated that the perceived duration of an empty interval, starting from the offset of a first marker and ending with the onset of a second marker, depended on the size of the markers that demarcated the empty interval. Specifically, empty intervals that were sand-
A

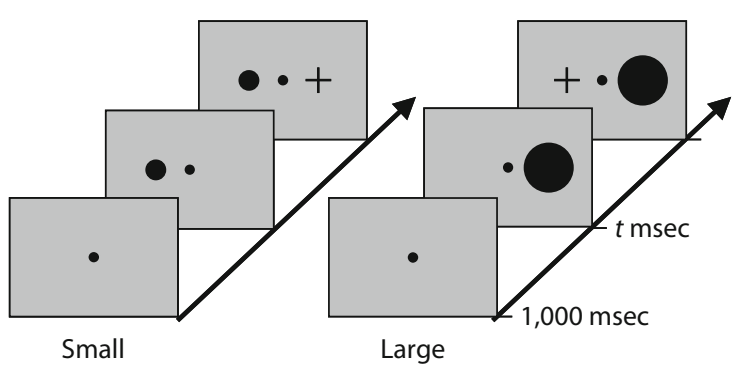

B

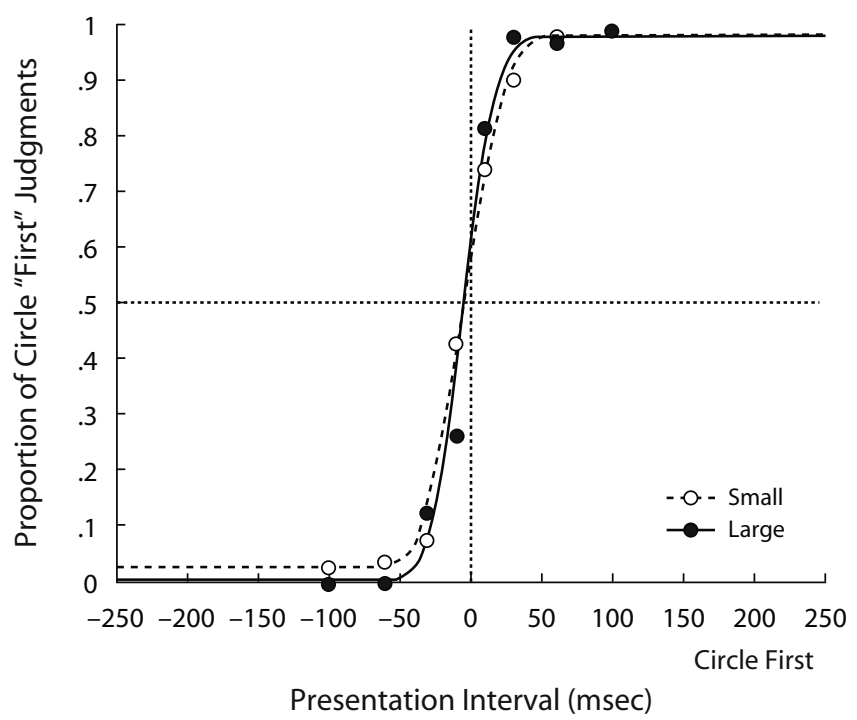

Figure 3. Effects of the marker size on temporal-order judgments (Experiment 7). (A) Participants judged the order of two visual stimuli (a cross and a filled circle) that were presented in succession. The filled circle was small under one condition (small) and large under another (large). Participants were required to fixate on the center target. (B) The order-judgment probability that the circle was presented earlier than the cross is plotted against the presentation interval. Positive intervals show that the circle was presented first. Each symbol represents 80 judgments from 8 participants. 
wiched between the presentation of large stimuli were experienced as longer in duration than those that were sandwiched between the presentation of small stimuli; this effect depended on the size of the second marker in particular. These findings represent the first research that shows any influence of nontemporal marker properties on perceptions of empty intervals.

\section{Relationship With Previous Hypotheses}

First, we discuss whether our results can be explained by the internal-marker hypothesis. As noted above, this hypothesis predicts that the perceived duration should be smaller with larger markers than with smaller markers. In a clear departure from this prediction, our study showed that the perceived duration of the empty interval became longer in the presence of larger markers. These results might be reconciled with those of the internalmarker hypothesis if the detection of the larger marker was delayed relative to that of the smaller marker. Results of our final experiments clearly excluded this possibility, however, by showing that detection of the larger marker was as fast as detection of the smaller marker. We conclude, therefore, that our results cannot be explained by the internal-marker hypothesis.

Other studies have suggested that experience of the duration of an empty interval starts from the onset, rather than from the offset, of the first marker, and that it ends with the onset of the second marker (the onset-onset hypothesis; Grondin et al., 1996; Woodrow, 1928). According to the onset-onset hypothesis, the perceived duration of an empty interval should depend on the duration of the first marker; however, the perceived duration changed even when the duration of the first marker was kept constant $(1.5 \mathrm{sec}$; Experiments 1-3). In addition, the dominant factor in the markersize effect involved the properties of the second marker, rather than those of the first marker; thus, our results cannot be explained by the onset-onset hypothesis.

Studies on visible persistence have shown that manipulating the intensity of a display sometimes produces an inverse-intensity effect (Allport, 1968; Di Lollo \& Bischof, 1995): The visible persistence remains as the intensity of the visual stimuli decreases. That is, contrary to predictions that are derived from time-perception studies (Long \& Beaton, 1980; Nisly \& Wasserman, 1989; Thomas \& Cantor, 1975), the inverse-intensity effect predicts that the perceived offset of a small first marker will be more delayed than that of a large marker. The inverse-intensity effect, therefore, seemingly accounts for our finding that the duration of time that was experienced in viewing an empty interval that was sandwiched between small stimuli was shorter than the duration of time that was experienced in viewing an empty interval that was sandwiched between large stimuli. If our findings regarding the marker-size effect were caused by the inverse-intensity effect, the perceived duration of the empty interval should have depended primarily on the intensity of the first marker; however, the properties of the second marker represented the dominant factors in the marker-size effect. Thus, the present findings cannot be explained by the inverse-intensity effect.

\section{Effects of the Second Marker}

Previous studies on interval perception (Gibbon, 1991; Treisman, 1963) have hypothesized that an internal clock generates pulses that are gated by a switch that is turned on and off by time markers; these markers are presumed to indicate the beginning and the end of an interval. These pulses are then "counted" while the switch is closed, and this pulse count is stored in working memory. This count represents the experienced duration of the interval. As discussed above, however, the pulse count between the two time markers does not explain our results.

We propose instead that the signals that are elicited by the second marker modify the working memory in which the pulse count is stored. This postdictive framework is consistent with previous findings regarding vision, such as backward masking (Bachmann, 1994), the color-phi phenomenon (Kolers \& von Grünau, 1976), and the flashlag effect (Eagleman \& Sejnowski, 2000). These previous studies have shown that a visual stimulus that was presented later in time blocked or modified the perception of preceding events. The memory of the pulse count, therefore, was likely modified by the signals of the second marker before the pulse count accounted for the perception of an empty interval. Our hypothesis also agrees with more recent hypotheses regarding experiences of timing that emphasize the importance of working memory (d'Avossa et al., 2007; Johnston, Arnold, \& Nishida, 2006; Lewis \& Miall, 2006; Morrone, Ross, \& Burr, 2005; Staddon, 2005).

The foregoing discussion begs the question: How do these modifications vary depending on the size of markers? In Experiment 4, we excluded the possibility that participants had added the entire second-marker duration into their experience of the time that was spent viewing the preceding empty interval. Nonetheless, participants may have added a small initial period adhering to the second marker presentation. Even if this were the case, however, the amount of addition was correlated with the size of the second marker (i.e., the larger the marker, the longer the perceived interval). The results clearly show that the hypothesized addition of the initial part of the second marker was not caused "inadvertently" but in a more systematic way.

Finally, the marker-size effect (the larger, the longer) was specific to the empty interval, and it was reversed when the empty interval was filled with the presentation of a red circle (Figure 1I). These results suggest that there are two distinct mechanisms that are pertinent to experiences of elapsed time: one for filled intervals and another for empty intervals. Although we cannot dismiss the possibility that a single mechanism reacts differently to these inputs, these data are consistent with recent findings in neurophysiology that there are two groups of neurons that might contribute to such timing processes. The activities of neurons in one group increase in a linear manner, whereas those in the other group decrease in an exponential manner (Lewis \& Miall, 2006; Staddon, 2005). Neurons in the first group have been found in the lateral intraparietal area (Janssen \& Shadlen, 2005), the supplementary eye field (Ohmae, Lu, Takahashi, Uchida, \& Kitazawa, 2008) and 
the dorsolateral prefrontal cortex (Fuster, 2001). Neurons in the second group have been found in the dorsolateral prefrontal cortex (Fuster, 2001). A recent neuroimaging study in humans reported that subregions in the dorsolateral prefrontal cortex increased their average activity as the presented interval increased, whereas other subregions decreased their activity, supporting the idea that both increasing and decaying activity could serve as measures of time (Jech, Dušek, Wackermann, \& Vymazal, 2005; Lewis $\&$ Miall, 2006). We still do not know how or whether these two groups of neurons contribute to timing processes; however, we speculate that one group of neurons plays a major role in the timing process for the filled interval, and the other plays a major role for the empty interval. We believe that this hypothesis warrants further testing.

\section{AUTHOR NOTE}

The study was partly supported by Grants-in-Aid for Scientific Research (A) 18200024 from the Japan Society for the Promotion of Science to S.K., and by a grant from the Research Fellowships of the Japan Society for the Promotion of Science for Young Scientists to F.O. Correspondence concerning this article should be addressed to F. Ono, Department of Neurophysiology, Juntendo University School of Medicine, 2-1-1 Hongo, Bunkyo-ku, Tokyo 113-8421, Japan (e-mail: fuminori@ juntendo.ac.jp).

\section{REFERENCES}

Allport, D. A. (1968). Phenomenal simultaneity and the perceptual moment hypothesis. British Journal of Psychology, 59, 395-406.

BaChmann, T. (1994). Psychophysiology of visual masking: The fine structure of conscious experience. New York: Nova Science.

Brainard, D. H. (1997). The Psychophysics Toolbox. Spatial Vision, 10, 433-436.

Cooper, L. A., \& Weintraub, D. J. (1970). Delboeuf-type circle illusions: Interactions among luminance, temporal characteristics, and inducing-figure variations. Journal of Experimental Psychology, 85, 75-82.

D’Avossa, G., Tosetti, M., Crespi, S., Biagi, L., Burr, D. C., \& MorRONE, M. C. (2007). Spatiotopic selectivity of BOLD responses to visual motion in human area MT. Nature Neuroscience, 10, 249-255.

Di Lollo, V., \& BischOF, W. F. (1995). Inverse-intensity effect in duration of visible persistence. Psychological Bulletin, 118, 223-237.

Eagleman, D. M., \& SeJnowski, T. J. (2000). Motion integration and postdiction in visual awareness. Science, 287, 2036-2038.

Fuster, J. M. (2001). The prefrontal cortex - an update: Time is of the essence. Neuron, 30, 319-333.

GibBon, J. (1991). Origins of scalar timing. Learning \& Motivation, 22, 3-38.

Grondin, S. (1993). Duration discrimination of empty and filled intervals marked by auditory and visual signals. Perception \& Psychophysics, 54, 383-394.

Grondin, S., Ivry, R. B., Franz, E., Perreault, L., \& Metthé, L.
(1996). Markers' influence on the duration discrimination of intermodal intervals. Perception \& Psychophysics, 58, 424-433.

JANSSEN, P., \& ShadLEN, M. N. (2005). A representation of the hazard rate of elapsed time in macaque area LIP. Nature Neuroscience, $\mathbf{8}$, 234-241.

Jech, R., Dušek, P., Wackermann, J., \& Vymazal, J. (2005). Cumulative blood oxygenation-level-dependent signal changes support the 'time accumulator' hypothesis. NeuroReport, 16, 1467-1471.

Johnston, A., ARNOLD, D. H., \& NishidA, S. (2006). Spatially localized distortions of event time. Current Biology, 16, 472-479.

Kolers, P. A., \& vON GrÜNAU, M. (1976). Shape and color in apparent motion. Vision Research, 16, 329-335.

Lewis, P. A., \& Miall, R. C. (2006). Remembering the time: A continuous clock. Trends in Cognitive Sciences, 10, 401-406.

Long, G. M., \& BEATON, R. J. (1980). The contribution of visual persistence to the perceived duration of brief targets. Perception \& Psychophysics, 28, 422-430.

Mo, S. S. (1975). Temporal reproduction of duration as a function of numerosity. Bulletin of the Psychonomic Society, 5, 165-167.

Morrone, M. C., Ross, J., \& BURR, D. (2005). Saccadic eye movements cause compression of time as well as space. Nature Neuroscience, $\mathbf{8}$, 950-954.

NisLy, S. J., \& Wasserman, G. S. (1989). Intensity dependence of perceived duration: Data, theories, and neural integration. Psychological Bulletin, 106, 483-496.

Ohmae, S., Lu, X., Takahashi, T., Uchida, Y., \& Kitazawa, S. (2008). Neuronal activity related to anticipated and elapsed time in macaque supplementary eye field. Experimental Brain Research, 184, 593-598.

Ono, F., \& KaWAhara, J.-I. (2007). The subjective size of visual stimuli affects the perceived duration of their presentation. Perception \& Psychophysics, 69, 952-957.

PiÉRON, H. (1952). The sensations: Their functions, processes, and mechanisms (M. H. Pirenne \& B. C. Abbott, Trans.). New Haven, CT: Yale University Press.

Pins, D., \& Bonnet, C. (1996). On the relation between stimulus intensity and processing time: Piéron's law and choice reaction time. Perception \& Psychophysics, 58, 390-400.

Schiffman, H. R., \& BobKo, D. J. (1974). Effects of stimulus complexity on the perception of brief temporal intervals. Journal of Experimental Psychology, 103, 156-159.

Staddon, J. E. R. (2005). Interval timing: Memory, not a clock. Trends in Cognitive Sciences, 9, 312-314.

StAFFord, T., \& GuRNEY, K. N. (2004). The role of response mechanisms in determining reaction time performance: Piéron's law revisited. Psychonomic Bulletin \& Review, 11, 975-987.

Thomas, E. A. C., \& CANTOR, N. E. (1975). On the duality of simultaneous time and size perception. Perception \& Psychophysics, 18, 44-48.

Treisman, M. (1963). Temporal discrimination and the indifference interval: Implications for a model of the "internal clock." Psychological Monographs, 77, 1-31.

Tse, C.-Y., \& Penney, T. B. (2006). Preattentive timing of empty intervals is from marker offset to onset. Psychophysiology, 43, 172-179.

Woodrow, H. (1928). Behavior with respect to short temporal stimulusforms. Journal of Experimental Psychology, 11, 167-193.

(Manuscript received October 28, 2007; revision accepted for publication July 20, 2008.) 